\title{
Pacific
}

Journal of

Mathematics

\section{ON DIVISORS OF SUMS OF INTEGERS. V}

ANDRÁS SÁRKÖZY AND CAMERON LEIGH STEWART 


\section{ON DIVISORS OF SUMS OF INTEGERS $V$}

\section{A. SÁrKözy AND C. L. STEWART}

Dedicated to Professor P. Erdős on the occasion of his eightieth birthday.

Let $N$ be a positive integer and let $A$ and $B$ be subsets of $\{1, \ldots, N\}$. In this article we shall estimate both the maximum and the average of $\omega(a+b)$, the number of distinct prime factors of $a+b$, where $a$ and $b$ are from $A$ and $B$ respectively.

1. Introduction. For any set $X$ let $|X|$ denote its cardinality and for any integer $n$ larger than one let $\omega(n)$ denote the number of distinct prime factors of $n$. Let $I$ be an integer larger than one and let $\epsilon$ be a positive real number. Let $2=p_{1}, p_{2}, \ldots$ be the sequence of prime numbers in increasing order and let $m$ be that positive integer for which $p_{1} \cdots p_{m} \leq N \leq p_{1} \cdots p_{m+1}$. In [3], Erdős, Pomerance, Sárközy and Stewart proved that there exist positive numbers $C_{0}$ and $C_{1}$ which are effectively computable in terms of $\epsilon$, such that if $N$ exceeds $C_{0}$ and $A$ and $B$ are subsets of $\{1, \ldots, N\}$ with $(|A||B|)^{1 / 2}>\epsilon N$ then there exist integers $a$ from $A$ and $b$ from $B$ for which

$$
\omega(a+b)>m-C_{1} \sqrt{m} .
$$

They also showed that there is a positive real number $\epsilon$, with $\epsilon<1$, and an effectively computable positive number $C_{2}$ such that for each positive integer $N$ there is a subset $A$ of $\{1, \ldots, N\}$ with $|A| \geq \epsilon N$ for which

$$
\max _{a, a^{\prime} \in A} \omega\left(a+a^{\prime}\right)<m-\frac{C_{2} \sqrt{m}}{\log m} .
$$

Notice by the prime number theorem that

$$
m=(1+o(1))(\log N) /(\log \log N) .
$$


In this article we shall study both the maximum of $\omega(a+b)$ and the average of $\omega(a+b)$ as $a$ and $b$ run over $A$ and $B$ respectively where $A$ and $B$ are subsets of $\{1, \ldots, N\}$ for which $(|A||B|)^{1 / 2}$ is much smaller than $\epsilon N$. Our principal tool will be the large sieve inequality.

THEOREM 1. Let $\theta$ be a real number with $1 / 2<\theta \leq 1$ and let $N$ be a positive integer. There exists a positive number $C_{3}$, which is effectively computable in terms of $\theta$, such thet if $A$ and $B$ are subsets of $\{1, \ldots, N\}$ with $N$ greater than $C_{3}$ and

$$
(|A||B|)^{1 / 2} \geq N^{\theta}
$$

then there exists an integer a from $A$ and an integer $b$ from $B$ for which

$$
\omega(a+b)>\frac{1}{6}\left(\theta-\frac{1}{2}\right)^{2}(\log N) / \log \log N
$$

In [6] Pomerance, Sárközy and Stewart showed that if $A$ and $B$ are sufficiently dense sets then there is a sum $a+b$ which is divisible by a small prime factor. In particular they proved the following result. Let $\beta$ be a positive real number. There is a positive number $C_{4}$, which is effectively computable in terms of $\beta$, such that if $A$ and $B$ are subsets of $\{1, \ldots, N\}$ with $(|A||B|)^{1 / 2}>C_{4} N^{1 / 2}$ then there is a prime number $p$ with $\beta<p<C_{4}\left(N /(|A||B|)^{1 / 2}\right)$, an integer $a$ from $A$ and an integer $b$ from $B$ such that $p$ divides $a+b$. As a byproduct of our proof of Theorem 1 we are able to improve upon this result.

THEOREM 2. Let $N$ be a positive integer and let $\theta$ and $\beta$ be real numbers with $1 / 2<\theta<1$. There is a positive number $C_{5}$, which is effectively computable in terms of $\theta$ and $\beta$, such that if $A$ and $B$ are subsets of $\{1, \ldots, N\}$ with

$$
(|A||B|)^{1 / 2} \geq N^{\theta}
$$

and $N$ exceeds $C_{5}$ then there is a prime number $p$ with

$$
\beta<p \leq\left(\frac{\log N}{2}\right)^{1 /(2 \theta-1)}
$$


such that every residue class modulo $p$ contains a member of $A+B$.

It follows from the work of Elliott and Sárközy [1], see also Erdős, Maier and Sárközy [2] and Tenenbaum [7], that if $A$ and $B$ are subsets of $\{1, \ldots, N\}$ with

$$
(|A||B|)^{1 / 2}=N / \exp \left(o\left((\log \log N)^{1 / 2} \log \log \log N\right)\right)
$$

and $N$ is sufficiently large then a theorem of Erdoss-Kac type holds for $\omega(a+b)$. In particular for $A$ and $B$ satisfying (4) we have

$$
\frac{1}{|A||B|} \sum_{a \in A} \sum_{b \in B} \omega(a+b) \sim \log \log N .
$$

Let $\delta$ be a positive real number. If $A$ and $B$ are subsets of $\{1, \ldots, N\}$ with $|A| \sim|B| \sim N \exp (-\delta \log \log \log N)$, then (5) need not hold. For instance we may take $A$ and $B$ to be the subset of $\{1, \ldots, N\}$ consisting of the multiples of $\prod_{p<\delta \log \log N \log \log \log N} p$. Then for $N$ sufficiently large the average of $\omega(a+b)$ is at least $(1+\delta / 2) \log \log N$. On the other hand we conjecture that if $A$ and $B$ are subsets of $\{1, \ldots, N\}$ with

$$
\min (|A|,|B|)>\exp \left((\log N)^{1+o(1)}\right)
$$

$\epsilon$ is a positive real number and $N$ is sufficiently large in terms of $\epsilon$ then

$$
\frac{1}{|A||B|} \sum_{a \in A} \sum_{b \in B} \omega(a+b)>(1-\epsilon) \log \log N .
$$

On taking $A$ and $B$ to be positive integers up to $\exp \left((\log N)^{1-\epsilon}\right)$ we see that condition (6) cannot be weakened substantially. Furthermore, we conjecture that if we let $N$ tend to infinity and $A$ and $B$ run over subsets of $\{1, \ldots, N\}$ with

$$
\frac{\log (\min (|A|,|B|))}{\log \log N} \rightarrow \infty
$$

then

$$
\frac{1}{|A||B|} \sum_{a \in A} \sum_{b \in B} \omega(a+b) \rightarrow \infty
$$


While we have not been able to establish (7) for all subsets $A$ and $B$ satisfying (6), we have been able to determine the average order for the number of large prime divisors of the sums $a+b$ for sufficiently dense sets $A$ and $B$. As a consequence we are able to establish (7) for such sets.

THEOREM 3. There exists an effectively computable positive constant $C_{6}$ such that if $T$ and $N$ are positive integers with $T \leq \sqrt{2 N}$ and $A$ and $B$ are non-empty subsets of $\{1, \ldots, N\}$ then

$$
\begin{aligned}
\mid \frac{1}{|A||B|} \sum_{T<p} \sum_{a \in A, b \in B, p \mid(a+b)} 1-(\log \log N- & \log \log (3 T)) \mid \\
& <C_{6}+\frac{3 N}{(|A||B|)^{1 / 2} T} .
\end{aligned}
$$

We now take $T=N /(|A||B|)^{1 / 2}$ in Theorem 3 to obtain the following result.

COROLlaRY 1. There exists an effectively computable positive constant $C_{7}$ such that if $N$ is a positive integer and $A$ and $B$ are subsets of $\{1, \ldots, N\}$ with $|A||B|>N$ then

$$
\begin{aligned}
\mid \frac{1}{|A||B|} \sum_{p>N(|A||B|)^{-1 / 2}} \sum_{a \in A, b \in B, p \mid(a+b)} & 1-(\log \log N \\
& \left.-\log \log N(|A||B|)^{1 / 2}\right) \mid<C_{7} .
\end{aligned}
$$

Therefore (7) holds for $N$ sufficiently large provided that $A$ and $B$ are subsets of $\{1, \ldots, N\}$ with

$$
(|A||B|)^{1 / 2}=N \exp \left((\log N)^{o(1)}\right) .
$$

2. Preliminary Lemmas. For any real number $x$ let $e(x)=$ $e^{2 \pi i}$ and let $\|x\|$ denote the distance from $x$ to the nearest integer. 
Let $M$ and $N$ be integers with $N$ positive and let $a_{M+1}, \ldots, a_{M+N}$ be complex numbers. Define $S(x)$ by

$$
S(x)=\sum_{M+1}^{M+N} a_{n} e(n x) .
$$

Let $X$ be a set of real numbers which are distinct modulo 1 and define $\delta$ by

$$
\delta=\min _{x, x^{\prime} \in X, x \neq x^{\prime}}\left\|x-x^{\prime}\right\|
$$

The analytical form of the large sieve inequality, (see Theorem 1 of [5]), is required for the proof of Theorem 3 and it is given below.

LEMMA 1. Let $S(x)$ and $\delta$ be as in (8) and (9), respectively. Then

$$
\sum_{x \in X}|S(x)|^{2} \leq\left(N+\delta^{-1}\right) \sum_{n=M+1}^{M+N}\left|a_{n}\right|^{2} .
$$

We shall also make use of the following result, see Theorem 1 of [6], which was deduced with the aid of the arithmetical form of the large sieve inequality.

Lemma 2. Let $N$ be a positive integer and let $A$ and $B$ be nonempty subsets of $\{1, \ldots, N\}$. Let $S$ be a set of prime numbers, let $Q$ be a positive integer and let $J$ denote the number of square-free positive integers up to $Q$ all of whose prime factors are from $S$. If

$$
J(|A||B|)^{1 / 2}>N+Q^{2}
$$

then there is a prime $p$ in $S$ such that each residue class modulo $p$ contains a member of the sum set $A+B$.

Finally, to prove Theorems 1 and 2 we shall require the next result.

LEMMA 3. Let $\alpha$ and $\beta$ be real numbers with $\alpha>1$ and let $N$ be a positive integer. Let $T$ be the set of prime numbers $p$ which satisfy $\beta<p \leq(\log N)^{\alpha}$ and let $S$ be a subset of $T$ consisting of all but 
at most $2 \log N$ elements of $T$. Let $R$ denote the set of square-free positive integers less than or equal to $N$ all of whose prime factors are from $S$. There exists a real number $C_{8}$, which is effectively computable in terms of $\alpha$ and $\beta$, such that

$$
|R|>20 N^{1-1 / \alpha}
$$

whenever $N$ is greater then $C_{8}$.

Proof. $C_{9}, C_{10}$ and $C_{11}$ will denote positive numbers which are effectively computable in terms of $\alpha$ and $\beta$. By the prime number theorem with error term,

$$
|S| \geq \pi\left((\log N)^{\alpha}\right)-\pi(\beta)-2 \log N>\frac{(\log N)^{\alpha}}{\alpha \log \log N},
$$

provided that $N$ is greater than $C_{9}$. For any real number $x$ let $[x]$ denote the greatest integer less than or equal to $x$. We now count the number of distinct ways of choosing $[\log N /(\alpha \log \log N)]$ primes from $S$. Each choice gives rise to a distinct square-free integer, given by the product of the primes, which does not exceed $N$ and is composed only of primes from $S$. Then $|R| \geq \omega$ where

$$
\omega=\left(\left[\frac{|S|}{\log N}\right]\right) .
$$

Thus

$$
\omega \geq \frac{\left(|S|-\left[\frac{\log N}{\alpha \log \log N}\right]\right)^{\frac{\log N}{\alpha \log \log N}-1}}{\left[\frac{\log N}{\alpha \log \log N}\right] !}
$$

and so, by (11) and Stirling's formula,

$$
\omega \geq \frac{\left(\frac{(\log N)^{\alpha}}{\alpha \log \log N}\left(1-\frac{1}{(\log N)^{\alpha-1}}\right)\right)^{\frac{\log N}{\alpha \log \log N}}}{(\log N)^{\alpha+1}\left(\frac{\log N}{e \alpha \log \log N}\right)^{\frac{\log N}{\alpha \log \log N}}}
$$


for $N>C_{10}$. Since $\log (1-x)>-2 x$ for $0<x<1 / 2$, we find that, for $N>C_{11}$,

$$
\omega \geq N^{1-1 / \alpha} e^{\left(\frac{\log N}{\alpha \log \log N}-\frac{2(\log N)^{2-\alpha}}{\alpha \log \log N}\right)}(\log N)^{-\alpha-1},
$$

hence

$$
\omega>20 N^{1-1 / \alpha},
$$

as required.

3. Proof of Theorem 1. Let $\theta_{1}=(\theta+1 / 2) / 2$ and define $G$ and $v$ by

$$
G=(\log N)^{1 /\left(2 \theta_{1}-1\right)},
$$

and

$$
v=\left[\frac{1}{6}\left(\theta-\frac{1}{2}\right)^{2} \frac{\log N}{\log \log N}\right]+1,
$$

respectively.

Put $A_{0}=A, B_{0}=B$ and $W_{0}=\emptyset$. We shall construct inductively sets $A_{1}, \ldots, A_{v}, B_{1}, \ldots, B_{v}$ and $W_{1}, \ldots, W_{v}$ with the following properties. First, $W_{i}$ is a set of $i$ primes $q$ satisfying $10<q \leq G, A_{i} \subseteq$ $A_{i-1}$ and $B_{i} \subseteq B_{i-1}$ for $i=1, \ldots, v$. Secondly every element of the sum set $A_{i}+B_{i}$ is divisible by each prime in $W_{i}$ for $i=1, \ldots, v$. Finally,

$$
\left|A_{i}\right| \geq \frac{|A|}{G^{3 i}} \quad \text { and } \quad\left|B_{i}\right| \geq \frac{|B|}{G^{3 i}},
$$

for $i=1, \ldots, v$. Note that this suffices to prove our result since $A_{v}$ and $B_{v}$ are both non-empty and on taking $a$ from $A_{v}$ and $b$ from $B_{v}$ we find that $a+b$ is divisible by the $v$ primes from $W_{v}$ and so (2) follows from (12).

Suppose that $i$ is an integer with $0 \leq i<v$ and that $A_{i}, B_{i}$ and $W_{i}$ have been constructed with the above properties. We shall now show how to construct $A_{i+1}, B_{i+1}$ and $W_{i+1}$. First, for each prime $p$ with $10<p \leq G$ let $a_{1}, \ldots, a_{j(p)}$ be representatives for those residue classes modulo $p$ which are occupied by fewer than $\left|A_{i}\right| / p^{3}$ terms of $A_{i}$. For each prime $p$ with $10<p \leq G$ we remove from $A_{i}$ those 
terms of $A_{i}$ which are congruent to one of $a_{1}, \ldots, a_{j(p)}$ modulo $p$. We are left with a subset $A_{i}^{\prime}$ of $A_{i}$ with

(14) $\left|A_{i}^{\prime}\right| \geq\left|A_{i}\right|\left(1-\sum_{10<p \leq G} \frac{j(p)}{p^{3}}\right) \geq\left|A_{i}\right|\left(1-\sum_{10<p} \frac{1}{p^{2}}\right) \geq \frac{\left|A_{i}\right|}{10}$

and such that for each prime $p$ with $10<p \leq G$ and each $a^{\prime}$ in $A_{i}^{\prime}$, the number of terms of $A_{i}$ which are congruent to $a^{\prime}$ modulo $p$ is at least $\left|A_{i}\right| / p^{3}$. Similarly, we produce a subset $B_{i}^{\prime}$ of $B_{i}$ with

$$
\left|B_{i}^{\prime}\right| \geq \frac{\left|B_{i}\right|}{10}
$$

and such that for each prime $p$ with $10<p \leq G$ and each residue class modulo $p$ which contains an element of $B_{i}^{\prime}$ the number of terms of $B_{i}$ in the residue class is at least $\left|B_{i}\right| / p^{3}$.

The number of terms in $W_{i}$ is $i$ which is less than $v$ and, by (12), is at most $\log N$. Thus we may apply Lemma 3 with $\beta=10$ and $\alpha=1 /\left(2 \theta_{1}-1\right)$ to conclude that there is a real number $C_{12}$, which is effectively computable in terms of $\theta$, such that if $N$ exceeds $C_{12}$ then the number of square-free positive integers less than or equal to $N^{1 / 2}$ all of whose prime factors $p$ satisfy $10<p \leq G$ and $p \notin W_{i}$ is greater than

$$
20 N^{\frac{1}{2}\left(1-\left(2 \theta_{1}-1\right)\right)}=20 N^{1-\theta_{1}} .
$$

By our inductive assumption (13) and by (1) and (12), we obtain

$$
\left(\left|A_{i}\right|\left|B_{i}\right|\right)^{1 / 2} \geq(|A||B|)^{1 / 2} G^{-3 i} \geq N^{\theta_{1}} .
$$

Thus, by (14), (15) and (17),

$$
\left(\left|A_{i}^{\prime}\right|\left|B_{i}^{\prime}\right|\right)^{1 / 2} \geq \frac{N^{\theta_{1}}}{10}
$$

We now apply Lemma 2 with $A=A_{i}^{\prime}, B=B_{i}^{\prime}, Q=N^{1 / 2}$ and $S$ the set of primes $p$ with $10<p \leq G$ and $p \notin W_{i}$. Then $J$, the number of square-free integers up to $Q$ divisible only by primes from $S$, is greater than $20 N^{1-\theta_{1}}$ by (16), for $N>C_{12}$ and so, by (18), inequality (10) holds. Thus there is a prime $q_{i+1}$ in $S$, an element 
$a^{\prime}$ in $A_{i}^{\prime}$ and an element $b^{\prime}$ in $B_{i}^{\prime}$ such that $q_{i+1}$ divides $a^{\prime}+b^{\prime}$. We put

$$
\begin{aligned}
& A_{i+1}=\left\{a \in A_{i}: a \equiv a^{\prime}\left(\bmod q_{i+1}\right)\right\} \\
& B_{i+1}=\left\{b \in B_{i}: b \equiv b^{\prime}\left(\bmod q_{i+1}\right)\right\}
\end{aligned}
$$

and

$$
W_{i+1}=W_{i} \cup\left\{q_{i+1}\right\} .
$$

By our construction every element of $A_{i+1}+B_{i+1}$ is divisible by each prime in $W_{i+1}$. Further, we have, by (13),

$$
\left|A_{i+1}\right| \geq \frac{\left|A_{i}\right|}{q_{i+1}^{3}} \geq \frac{\left|A_{i}\right|}{G^{3}} \geq \frac{|A|}{G^{3(i+1)}}
$$

and

$$
\left|B_{i+1}\right| \geq \frac{|B|}{G^{3(i+1)}}
$$

as required. Our result now follows.

4. Proof of Theorem 2. Let $S$ be the set of primes $p$ which satisfy $\beta<p \leq\left(\log \left(N^{1 / 2}\right)\right)^{1 /(2 \theta-1)}$. Put $\alpha=1 /(2 \theta-1)$ and observe that $\alpha$ is a real number greater than one since $1 / 2<\theta<1$. Next let $J$ denote the number of square-free positive integer less than or equal to $N^{1 / 2}$ all of whose prime factors are from $S$. By Lemma 3 there exists a positive number $C_{13}$, which is effectively computable in terms of $\theta$, such that if $N$ exceeds $C_{13}$, then

$$
J>20\left(N^{1 / 2}\right)^{1-(2 \theta-1)}=20 N^{1-\theta} .
$$

We now apply Lemma 2 with $Q=N^{1 / 2}$ and with $J$ and $S$ as above. From (3) and (19) we obtain (10) and so our result follows from Lemma 2.

5. Proof of Theorem 3. Put $R=[\sqrt{2 N}]$. We have

$$
\begin{array}{rl}
\mid \sum_{a \in A} \sum_{b \in B} \sum_{T<p, p \mid a+b} & 1-\sum_{a \in A} \sum_{b \in B} \sum_{T<p \leq R, p \mid a+b} 1 \mid \\
= & \left|\sum_{a \in A} \sum_{b \in B} \sum_{R<p \leq 2 N, p \mid a+b} 1\right| \leq\left|\sum_{a \in A} \sum_{b \in B} 1\right|=|A||B| .
\end{array}
$$


We define, for each real number $\alpha$,

$$
F(\alpha)=\sum_{a \in A} e(a \alpha) \text { and } G(\alpha)=\sum_{b \in B} e(b \alpha) .
$$

Then

$$
\begin{gathered}
\sum_{a \in A} \sum_{b \in B} \sum_{T<p \leq R, p \mid a+b} 1=\sum_{T<p \leq R} \frac{1}{p} \sum_{h=0}^{p-1} F\left(\frac{h}{p}\right) G\left(\frac{h}{p}\right) \\
=\sum_{T<p \leq R} \frac{1}{p}\left(|A||B|+\sum_{h=0}^{p-1} F\left(\frac{h}{p}\right) G\left(\frac{h}{p}\right)\right) .
\end{gathered}
$$

Further there is an effectively computable positive constant $C_{14}$ such that

$$
\left|\sum_{T<p \leq R} \frac{1}{p}-(\log \log R-\log \log (3 T))\right|<C_{14},
$$

see Theorem 427 of [4]. Put

$$
H=\left|\sum_{a \in A} \sum_{b \in B} \sum_{T<p, p \mid a+b} 1-\right| A|| B|(\log \log N-\log \log (3 T))| .
$$

By (20), (21) and (22),

$$
H \leq C_{15}|A||B|+\sum_{T<p \leq R} \frac{1}{p} \sum_{h=1}^{p-1}\left|F\left(\frac{h}{p}\right) G\left(\frac{h}{p}\right)\right| .
$$

For all real numbers $u$ and $v,|u||v| \leq\left(|u|^{2}+|v|^{2}\right) / 2$ and thus

$$
\begin{array}{r}
H \leq C_{15}|A||B|+\frac{1}{2} \sum_{T<p \leq R} \frac{1}{p} \sum_{h=1}^{p-1}\left(\left(\frac{|B|}{|A|}\right)^{1 / 2}\left|F\left(\frac{h}{p}\right)\right|^{2}\right. \\
\left.+\left(\frac{|A|}{|B|}\right)^{1 / 2}\left|G\left(\frac{h}{p}\right)\right|^{2}\right) .
\end{array}
$$

Put

$$
S(n)=\sum_{p<n} \sum_{h=1}^{p-1}\left|F\left(\frac{h}{p}\right)\right|^{2}
$$


Then by Lemma 1 , for $n \leq R$,

$$
S(n) \leq\left(N+n^{2}\right)|A| \leq 3 N|A| .
$$

Thus we obtain

(24)

$$
\begin{aligned}
\sum_{T<p \leq R} \frac{1}{p} \sum_{h=1}^{p-1}\left|F\left(\frac{h}{p}\right)\right|^{2} \\
\quad=\sum_{n=T+1}^{R} \frac{S(n)-S(n-1)}{n} \\
=\sum_{n=T+1}^{R} S(n)\left(\frac{1}{n}-\frac{1}{n+1}\right)-\frac{S(T)}{T+1}+\frac{S(R)}{R+1} \\
=\sum_{n=T+1}^{R} 3 N|A|\left(\frac{1}{n}-\frac{1}{n+1}\right)+\frac{3 N|A|}{R+1}=\frac{3 N|A|}{T+1},
\end{aligned}
$$

and similarly

$$
\sum_{T<p \leq R} \frac{1}{p} \sum_{h=1}^{p-1}\left|G\left(\frac{h}{p}\right)\right|^{2} \leq \frac{3 N|B|}{T+1} .
$$

Our result follows from (23), (24) and (25).

\section{REFERENCES}

[1] P.D.T.A. Elliott, A. Sárközy, The distribution of the number of prime divisors of sums $a+b$, J. Number Theory, 29 (1988), 94-99.

[2] P. Erdős, H. Maier, A. Sárközy, On the distribution of the number of prime factors of sums $a+b$, Trans. Amer. Math. Soc., 302 (1987), 269-280.

[3] P. Erdős, C. Pomerance, A. Sárközy, C.L. Stewart, On elements of sumsets with many prime factors, J. Number Theory, 44 (1993), 93-104.

[4] G.H. Hardy, E.M. Wright, An introduction to the theory of numbers, 5-th ed., Oxford, 1979.

[5] H.L. Montgomery, R.C. Vaughan, The large seive, Mathematika, 20 (1973), 119-134.

[6] C. Pomerance, A. Sárközy, C.L. Stewart, On divisors of sums of integers, III, Pacific J. Math., 133 (1988), 363-379.

[7] G. Tenenbaum, Facteurs premiers de sommes d'entiers, Proc. Amer. Math. Soc., 106 (1989), 287-296. 
Received September 9, 1991, and accepted for publication July 29, 1993.

The research of the second author was supported in part by a Killam Research Fellowship and by Grant A3528 from the Natural Sciences and Engineering Research Council of Canada.

The University of WATERLOO

Waterloo, Ontario, Canada N2L 3G1

E-mail address: cstewart@watserv1.uwaterloo.ca

Permanent address of A. Sárközy:

Mathematical Institute

OF THE Hungarian ACADEMY OF SCIENCES

REÁLTANODA U. 13-15,

Budapest, Hungary, H-1053 



\section{PACIFIC JOURNAL OF MATHEMATICS}

Volume $166 \quad$ No. $2 \quad$ December 1994

Geometric aspects of Bäcklund transformations of Weingarten

submanifolds

STEVEN BUYSKE

Multipliers between invariant subspaces of the backward shift

225

ROBERT BRUCE CROFOOT

The Cauchy integral, analytic capacity and subsets of quasicircles

XIANG FANG

The number of lattice points within a contour and visible from the origin 295

Douglas Austin Hensley

On flatness of the Coxeter graph $E_{8}$

305

MASAKI IZUMI

Immersions up to joint-bordism

GUI SONG LI

Generalization of the Hilbert metric to the space of positive definite matrices

CARlangelo Liverani and Maciej WojtKowski

Periodicity, genera and Alexander polynomials of knots

SWATEE NAIK

On divisors of sums of integers. $\mathrm{V}$

ANDRÁS SÁRKÖZY and CAMERON LEIGH STEWART

Approximately inner automorphisms on inclusions of type $\mathrm{III}_{\lambda}$-factors

CARL WINSLøW

Correction to: "A convexity theorem for semisimple symmetric spaces"

KARL-HERMANN NEEB

Correction to: "Periodic points on nilmanifolds and solvmanifolds"

EDWARD KEPPELMANN

Correction to: "Partially measurable sets in measure spaces" 